\title{
パラメータ空間における最大シュール安定超球
}

\author{
小亀 英 已*・吉 田誠 一*・森 武 宏**
}

\section{The Largest Schur Stability Hypersphere in Parameter Space}

\author{
Hideki KOKAME*, Seiichi YoshidA* and Takehiro MoRI**
}

\begin{abstract}
For systems with uncertain parameters, stability robustness is often defined as the minimum of destabilizing parameter perturbation vectors, in the sense of a predescribed norm. The stability robustness thus defined can be visualized as the radius of the largest stability hypersphere in parameter space.

The present paper considers the stability robustness, assuming a characteristic polynomial whose coefficients are an affine function of real plant parameters. Explicit formulas are established for discretetime systems. They use two polynomials in the form of weighted sum of Chebyshev polynomials, determined from the coefficient data of the affine functions. Every hyperplane which consists of the boundary of stability region in the parameter space is found from the polynomials. Furthermore the explicit formulas would provide useful means to analyse continuity and discontinuity of the stability robustness with respect to the coefficient data.
\end{abstract}

Key Words : stability robustness, discrete-time system, affine parameters, stability hypersphere, Chebyshev polynomial

\section{1.はじめに}

不確定環境下にあるシステムの安定余裕（または安定 ロバスト度）を不確定性の構造を考慮した上で定義し， ロバスト設計に役立てようとする研究が近年盛んとなっ ている、特に不確定性の存在が系を記述するパラメータ に限定される場合には, 系をパラメータ空間内の 1 点と して表現し，その点を中心とする安定領域内部に含まれ る最大超球の半径をもって安定余裕と定義することが多 い.Soh ら ${ }^{1)}$ は特性多項式の係数空間を考え, ユークリッ ドノルムの意味で上記最大超球の半径を求める手順を与 えた. Biernācki ら ${ }^{2)}$ は特性多項式の係数が実パラメー 夕のアフィン関数となる場合を考え, 安定余裕を求める 同様の手順を与えた. 同じ問題に対するより具体的な計 算公式が Chapellat ら゙ によって与えられている。また

$*$ 大阪工業大学工学部 大阪市旭区大宮 5-16-1

** 京都工芸瀻維大学工芸学部 京都市左京区松ヶ崎御所海 道町

* Faculty of Engineering, Osaka Institute of Technology, Osaka

** Faculty of Engineering and Design, Kyoto Institute of Technology, Kyoto

(Received October 3, 1991)

(Revised January 17, 1992)

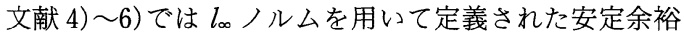
の計算法が示されている.一般に $l_{p}$ ノルムの場合に対し ては, 複素平面上のプロットから安定余裕を求める方法 が提案されている7),8

ところで文献 1)～3）で扱われたユークリッドノルム による場合, 安定余裕の計算はある有理関数の最小値を 求めることに帰着する．離散時間系の場合に同種の結果 を導く試みが Aguirre ら その関数形を具体的に示すまでには至っていない. そこ で本報告では，その関数がチェビシェフ多項式を用いて 簡潔な形に表現できることを示す。さらにアフィンパラ メータの場合では係数パラメータと異なり, パラメータ 空間中で安定領域の境界が 3 個以上の超平面を含む場合 が生じるが，この検証は同関数の分子多項式の根を調べ ることで容易に行われることを示す。

本文中, $\|\cdot\|$ でユークリッドノルムを表わす.また行列 $\boldsymbol{A}$ に対して, $\boldsymbol{A}\left(\begin{array}{ll}i_{1} & i_{2} \\ j_{1} & j_{2}\end{array}\right)$ で $\boldsymbol{A}$ の $i_{1}, i_{2}$ 行と $j_{1}, j_{2}$ 列を抜きだ してできる $2 \times 2$ の部分行列の行列式を表わす.対称行列 $A$ に対して, $A>0$ は $\boldsymbol{A}$ が正定值であることを意味す る. 


\section{2. 主要な結果}

離散時間閉ループ系の特性多項式をつぎのように仮定 する.

$$
f(z, \boldsymbol{p})=z^{n}+a_{n-1}(\boldsymbol{p}) z^{n-1}+\cdots+a_{1}(\boldsymbol{p}) z+a_{0}(\boldsymbol{p})
$$

ここで $\boldsymbol{p}=\left[p_{1} \cdots p_{k}\right]^{T}$ は系を規定するパラメータのべク トルであり, 以後 $k \geq 2$ の場合を考える.また特性方程式 の係数はベクトル $\boldsymbol{p}$ のアフィン関数

$$
a_{i}(\boldsymbol{p})=a_{i 0}+a_{i 1} p_{1}+\cdots+a_{i k} p_{k}, \quad i=0,1, \cdots, n-1
$$

で与えられるものとする。

パラメータベクトルの公称值 $\boldsymbol{p}^{0}=\left[p_{1}^{0} \cdots p_{k}^{0}\right]^{T}$ において 系は安定, すなわち $f\left(z, \boldsymbol{p}^{0}\right)$ はシュール多項式であると して,この系の安定余裕をつぎの量によって定義しよう. $\rho=\sup _{r>0}\left\{r: \forall \boldsymbol{p} \in B_{r}\right.$ に対して $f(z, \boldsymbol{p})$ は安定 $\}$

ここで $B_{r}=\left\{\boldsymbol{p} \in R^{k}:\left\|\boldsymbol{p}-\boldsymbol{p}^{0}\right\| \leq r\right\}$ は $k$ 次元の実パラメ 一夕空間の中で, $\boldsymbol{p}^{0}$ を中心とする半径 $r$ の超球を表わす ことから, $\rho$ は $\boldsymbol{p}^{0}$ を中心とする最大安定超球の半径と呼 ばれる。

( 3 ) 式の条件部, すなわち $B_{r}$ が安定超球であるため の条件を零不包含原理 ${ }^{10)}$ を用いて書き直すと

$$
\begin{aligned}
\rho= & \sup _{r>0}\left\{r: f\left(e^{j \boldsymbol{\theta}}, \boldsymbol{p}\right) \neq 0, \forall\left\|\boldsymbol{p}-\boldsymbol{p}^{0}\right\| \leq r,\right. \\
& \forall \theta \in[0, \pi]\}
\end{aligned}
$$

あるいは

$$
\begin{aligned}
\rho= & \inf _{r>0}\left\{r: f\left(e^{j \theta}, \boldsymbol{p}\right)=0, \quad \exists\left\|\boldsymbol{p}-\boldsymbol{p}^{0}\right\| \leq r,\right. \\
& \exists \theta \in[0, \pi]\} \\
= & \inf _{r>0} \inf _{\theta \in[0, \pi]}\left\{r: f\left(e^{j \theta}, \boldsymbol{p}\right)=0, \quad \exists\left\|\boldsymbol{p}-\boldsymbol{p}^{0}\right\| \leq r\right\} \\
= & \inf _{\theta \in[0, \pi]} \rho(\theta)
\end{aligned}
$$

ただし

$$
\rho(\theta)=\inf _{r>0}\left\{r: f\left(e^{j \theta}, \boldsymbol{p}\right)=0, \quad \exists\left\|\boldsymbol{p}-\boldsymbol{p}^{0}\right\| \leq r\right\}
$$

$\rho(\theta)$ は点 $\boldsymbol{p}^{0}$ からパラメータ空間の閉集合

$$
\Pi(\theta)=\left\{\boldsymbol{p}: f\left(e^{j \theta}, \boldsymbol{p}\right)=0\right\}
$$

へのユークリッドノルムでの距離となることに注意す る.また $\Pi(\theta)=\emptyset$ (空集合)のときには $\rho(\theta)=+\infty$ と定 義して矛盾を生じない.

この時点で $\rho(\theta)$ の関数形は明らかでないが, $\theta$ の代 わりに $x=\cos \theta$ を変数として用いれば， $\rho$ の明示的な 表現を与えることが可能である。まず変数 $x$ の採用によ って $\theta \in[0, \pi]$ は $x \in[-1,1]$ と 1 対 1 に対応すること に注意しよう.したがって $\rho(\theta)$ の代わりに $\rho(x)$ などと 書いて混乱を生じない.たとえば

$$
\rho=\inf _{x \in[-1,1]} \rho(x)
$$

さて ( 7 )の条件をべクトル $\boldsymbol{p}$ についてまとめると

$$
f\left(e^{j \theta}, \boldsymbol{p}\right)=f_{0}\left(e^{j \theta}\right)+p_{1} f_{1}\left(e^{j \theta}\right)+\cdots+p_{k} f_{k}\left(e^{j \theta}\right)=0
$$

$$
\begin{gathered}
f_{i}(z)=\delta_{0 i} z^{n}+a_{n-1, i} z^{n-1}+\cdots+a_{1 i} z+a_{0 i}, \\
i=0,1, \ldots, k
\end{gathered}
$$

ただし $\delta_{0 i}$ はクロネッカのデルタである。（9)式を実部 と虚部に分け，その両式にそれぞれ

$$
\begin{aligned}
\cos i \theta & =T_{i}(\cos \theta)=T_{i}(x), \quad i=0,1,2, \ldots \\
\sin i \theta & =\sin \theta U_{i-1}(\cos \theta) \\
& =\sin \theta U_{i-1}(x), \quad i=1,2, \ldots
\end{aligned}
$$

を代入してつぎを得る。

$$
\begin{aligned}
\operatorname{Re} f\left(e^{j \theta}, \boldsymbol{p}\right)= & T_{n}(x)+\sum_{i=0}^{n-1} a_{i 0} T_{i}(x) \\
& +p_{1} \sum_{i=0}^{n-1} a_{i 1} T_{i}(x) \\
& +\cdots+p_{k} \sum_{i=0}^{n-1} a_{i k} T_{i}(x)=0
\end{aligned}
$$

$$
\begin{aligned}
\operatorname{Im} f\left(e^{j \theta}, \boldsymbol{p}\right)= & \sin \theta\left\{U_{n-1}(x)+\sum_{i=1}^{n-1} a_{i 0} U_{i-1}(x)\right. \\
& +p_{1} \sum_{i=1}^{n-1} a_{i 1} U_{i-1}(x) \\
& \left.+\cdots+p_{k} \sum_{i=1}^{n-1} a_{i k} U_{i-1}(x)\right\}=0
\end{aligned}
$$

ここで $T_{i}, U_{i}$ はそれぞれ第 1 種, 第 2 種チェビシェフ 多項式と呼ばれるもので

$$
\begin{aligned}
T_{i}(z)= & \cos (i \arccos z), \\
U_{i}(z)= & \sin ((i+1) \arccos z) / \sin \arccos z \\
& i=0,1, \ldots
\end{aligned}
$$

で定義される $i$ 次多項式である.

(12)，(13) 式は $\sin \theta \neq 0$, すなわち $x \in(-1,1)$ のとき つぎの行列表現の形にまとめられる。

$$
D A p+D a^{0}=0
$$

ただし

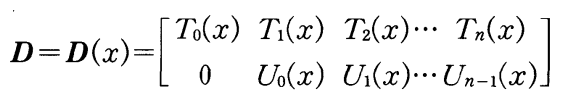$$
\in R^{2 \times(n+1)}
$$

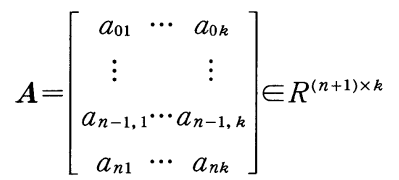

$\boldsymbol{a}^{0}=\left[\begin{array}{llll}a_{00} & a_{10} \cdots & a_{n-1,0} & a_{n 0}\end{array}\right]^{T} \in R^{n+1}$

また

$a_{n 1}=\cdots=a_{n k}=0, a_{n 0}=1$

に注意する。

$x=-1,1$ のとき $\operatorname{Im} f$ は常に零であるので, $f=0$ の条 件は

$$
\boldsymbol{d}_{1}^{T} \boldsymbol{A p}+\boldsymbol{d}_{1}^{T} \boldsymbol{a}^{0}=0
$$

となる．ここで $\boldsymbol{d}_{i}^{T}$ は $\boldsymbol{D}$ の第 $i$ 行べクトルを表わす。 
簡単のため,つぎの仮定が成り立つ場合を考える.

仮定 1. $\operatorname{rank} \boldsymbol{D}(x) \boldsymbol{A}=2,{ }^{\forall} x \in(-1,1)$

仮定 $2 . \boldsymbol{e}_{1}^{T} \boldsymbol{A} \neq 0, \boldsymbol{e}_{1}^{T}=[1 \cdots 1] \in R^{n+1}$

仮定 3. $\boldsymbol{e}_{2}^{T} \boldsymbol{A} \neq 0, \boldsymbol{e}_{2}^{T}=\left[\begin{array}{llll}1 & -1 & 1 & \cdots\end{array}(-1)^{n}\right] \in R^{n+1}$

この仮定が成立するときは, $\Pi(x)$ は $x=-1,+1$ に対し て $k-1$ 次元アフィン部分空間, また $x \in(-1,1)$ に対し て $k-2$ 次元アフィン部分空間をなす.このとき $\boldsymbol{p}^{0} を 中$ 心とする最大安定超球の半径 $\rho$ はつぎの定理によって 与えられる。

《定理 1》 仮定 $1,2,3$ の下で

$\rho=\min \left\{\mu_{1}, \mu_{2}, \bar{\rho}\right\}$

ただし

$$
\begin{aligned}
& \mu_{i}=\left|\boldsymbol{e}_{i}^{T} \boldsymbol{\delta}^{0}\right| /\left\|\boldsymbol{e}_{i}^{T} \boldsymbol{A}\right\|, \quad i=1,2 \\
& \boldsymbol{\delta}^{0}=\boldsymbol{A} \boldsymbol{p}^{0}+\boldsymbol{a}^{0}
\end{aligned}
$$

$\boldsymbol{\delta}^{0}$ は公称值 $\boldsymbol{p}^{0}$ での多項式 $f\left(z, \boldsymbol{p}^{0}\right)$ の係数べクトルを表 わす。また

$$
\begin{aligned}
& \bar{\rho}^{2}=\inf _{x \in(-1,1)} \rho^{2}(x) \\
& \rho^{2}(x)=\frac{\sum_{1 \leq l \leq k}\left\{n_{l}(x)\right\}^{2}}{\sum_{1 \leq l_{1}<l_{2} \leq k}\left\{d_{l_{1} l_{2}}(x)\right\}^{2}}
\end{aligned}
$$

ただし

$$
\begin{gathered}
d_{l_{1} l_{2}}(x)=\sum_{i=0}^{n-1} U_{i}(x) \sum_{j=1}^{n-i}\left(a_{j-1} l_{1} a_{j+i l_{2}}-a_{j-1 l_{2}} a_{j+i} l_{1}\right) \\
1 \leq l_{1}<l_{2} \leq k \\
n_{l}(x)=\sum_{i=0}^{n-1} U_{i}(x) \sum_{j=1}^{n-i}\left(a_{j-1} \delta_{l} \delta_{j+i}-a_{j+i}{ }_{l} \delta_{j-1}\right) \\
1 \leq l \leq k
\end{gathered}
$$

また

$$
\boldsymbol{\delta}^{0}=\left[\begin{array}{llll}
\delta_{0} & \delta_{1} & \cdots & \delta_{n}
\end{array}\right]^{T}
$$

(証明) はじめに( 6 )式は, $x \in[-1,1]$ として

$$
\rho(x)=\min \left\{\|\delta \boldsymbol{p}\|: f\left(e^{j \theta}, \boldsymbol{p}^{0}+\delta \boldsymbol{p}\right)=0\right\}
$$

と書き直されることに注意しておく.ここで $\delta \boldsymbol{p}=\boldsymbol{p}-\boldsymbol{p}^{0}$ は摂動ベクトルを表わす。

$$
\begin{gathered}
\boldsymbol{d}_{1}(1)=\boldsymbol{e}_{1}, \boldsymbol{d}_{1}(-1)=\boldsymbol{e}_{2} \text { より，(20)の条件はそれぞれ } \\
\boldsymbol{e}_{i}^{T} \boldsymbol{A} \delta \boldsymbol{p}+\boldsymbol{e}_{i}^{T} \boldsymbol{\delta}^{\mathbf{0}}=0, \quad i=1,2
\end{gathered}
$$

となる.これらの超平面の原点からの距離は (22)で与え られる. $x \in(-1,1)$ に対しては

$$
\boldsymbol{D A} \delta \boldsymbol{p}+\boldsymbol{D} \boldsymbol{\delta}^{0}=\mathbf{0}
$$

を得る。仮定 1 より，この連立方程式は解をもち，また その解の中で最小ノルムのものは, ムーア・ペンローズ の疑似逆行列を用いてつぎのように与えられる.

$$
\delta \boldsymbol{p}^{+}=-[\boldsymbol{D A}]^{+} \boldsymbol{D} \boldsymbol{\delta}^{0}
$$

また $D A \in R^{2 \times k}$ のランクは 2 であるから

$$
[\boldsymbol{D A}]^{+}=\boldsymbol{A}^{T} \boldsymbol{D}^{T}\left(\boldsymbol{D} \boldsymbol{A} \boldsymbol{A}^{T} \boldsymbol{D}^{T}\right)^{-1}
$$

で与えられる。ゆえに

$$
\rho^{2}(x)=\left\|\delta \boldsymbol{p}^{+}\right\|^{2}=\boldsymbol{\delta}^{0 T} \boldsymbol{D}^{T}\left(\boldsymbol{D} \boldsymbol{A} \boldsymbol{A}^{T} \boldsymbol{D}^{T}\right)^{-1} \boldsymbol{D} \boldsymbol{\delta}^{0}
$$

$$
\begin{aligned}
\operatorname{det} & \boldsymbol{D}\left[\boldsymbol{A} \boldsymbol{A}^{T}+\boldsymbol{\delta}^{0} \boldsymbol{\delta}^{0 T}\right] \boldsymbol{D}^{T} \\
= & \operatorname{det}\left[\boldsymbol{D} \boldsymbol{A} \boldsymbol{A}^{T} \boldsymbol{D}^{T}+\boldsymbol{D} \boldsymbol{\delta}^{0} \boldsymbol{\delta}^{0 T} \boldsymbol{D}^{T}\right] \\
= & \operatorname{det} \boldsymbol{D} \boldsymbol{A} \boldsymbol{A}^{T} \boldsymbol{D}^{T} \cdot \operatorname{det}[\boldsymbol{I} \\
& \left.+\left(\boldsymbol{D} \boldsymbol{A} \boldsymbol{A}^{T} \boldsymbol{D}^{T}\right)^{-1} \boldsymbol{D} \boldsymbol{\delta}^{0} \boldsymbol{\delta}^{0 T} \boldsymbol{D}^{T}\right] \\
= & \operatorname{det} \boldsymbol{D} \boldsymbol{A} \boldsymbol{A}^{T} \boldsymbol{D}^{T}\left(1+\boldsymbol{\delta}^{0 T} \boldsymbol{D}^{T}\left(\boldsymbol{D} \boldsymbol{A} \boldsymbol{A}^{T} \boldsymbol{D}^{T}\right)^{-1} \boldsymbol{D} \boldsymbol{\delta}^{0}\right) \\
= & \operatorname{det} \boldsymbol{D} \boldsymbol{A} \boldsymbol{A}^{T} \boldsymbol{D}^{T}\left(1+\rho^{2}(x)\right)
\end{aligned}
$$

より

$$
\rho^{2}(x)=\frac{\operatorname{det} \boldsymbol{D} \boldsymbol{B} \boldsymbol{B}^{T} \boldsymbol{D}^{T}}{\operatorname{det} \boldsymbol{D} \boldsymbol{A} \boldsymbol{A}^{T} \boldsymbol{D}^{T}}-1
$$

を得る、ただし

$$
\boldsymbol{B}=\left[\boldsymbol{A}: \boldsymbol{\delta}^{0}\right] \in R^{(n+1) \times(k+1)}
$$

である。ここで分母にコーシー・ビネーの公式を用いて

$$
d(x):=\operatorname{det} \boldsymbol{D} \boldsymbol{A} \boldsymbol{A}^{T} \boldsymbol{D}^{T}=\sum_{1 \leq l_{1}<l_{2} \leq k}\left\{d_{l_{1} l_{2}}(x)\right\}^{2}
$$

$$
d_{l_{1} l_{2}}(x)=[\boldsymbol{D A}]\left(\begin{array}{ll}
1 & 2 \\
l_{1} & l_{2}
\end{array}\right)
$$

さらに上式右辺に同公式を用いると

$$
d_{l_{1} l_{2}}(x)=\sum_{1 \leq j_{1}<j_{2} \leq n+1} \boldsymbol{D}\left(\begin{array}{ll}
1 & 2 \\
j_{1} & j_{2}
\end{array}\right) \boldsymbol{A}\left(\begin{array}{ll}
j_{1} & j_{2} \\
l_{1} & l_{2}
\end{array}\right)
$$

また(11)式の関係を用いて

$$
\begin{aligned}
& \boldsymbol{D}\left(\begin{array}{cc}
1 & 2 \\
i+1 & j+1
\end{array}\right)=\left|\begin{array}{cc}
T_{i}(x) & T_{j}(x) \\
U_{i-1}(x) & U_{j-1}(x)
\end{array}\right| \\
& =T_{i}(x) U_{j-1}(x)-T_{j}(x) U_{i-1}(x) \\
& =\cos i \theta\left(\frac{\sin j \theta}{\sin \theta}\right)-\cos j \theta\left(\frac{\sin i \theta}{\sin \theta}\right) \\
& =\sin (j-i) \theta / \sin \theta \\
& =U_{j-i-1}(x), \quad 0 \leq i<j
\end{aligned}
$$

そこで $\boldsymbol{D}\left(\begin{array}{ll}1 & 2 \\ j_{1} & j_{2}\end{array}\right)$ は添え字 $j_{2}, j_{1}$ の差にのみ依存すること に注目して $i=j_{2}-j_{1}-1$ として整理すると (26) を得る.

(35)の分子については

$$
\begin{aligned}
\tilde{n}(x):= & \operatorname{det} \boldsymbol{D} \boldsymbol{B} \boldsymbol{B}^{T} \boldsymbol{D}^{T} \\
= & \sum_{1 \leq l_{1}<l_{2} \leq k+1}\left\{[\boldsymbol{D} \boldsymbol{B}]\left(\begin{array}{ll}
1 & 2 \\
l_{1} & l_{2}
\end{array}\right)\right\}^{2} \\
= & \sum_{1 \leq l_{1}<l_{2} \leq k}\left\{[\boldsymbol{D A}]\left(\begin{array}{ll}
1 & 2 \\
l_{1} & l_{2}
\end{array}\right)\right\}^{2} \\
& +\sum_{1 \leq l \leq k} \operatorname{det}\left[\boldsymbol{D} \boldsymbol{A}_{l}\right]^{2}
\end{aligned}
$$

を得る。ただし $\boldsymbol{A}_{l}=\left[\boldsymbol{a}_{l}: \boldsymbol{\delta}^{0}\right] \in R^{(n+1) \times 2}$ ，また $\boldsymbol{a}_{l}$ は $\boldsymbol{A}$ の 第 $l$ 列べクトルである.ここで

$$
\begin{aligned}
& n_{l}(x)=\operatorname{det}\left[\boldsymbol{D} \boldsymbol{A}_{l}\right] \\
& n(x)=\sum_{1 \leq l \leq k}\left\{n_{l}(x)\right\}^{2}
\end{aligned}
$$

とおくと，(41)式は

$$
\tilde{n}(x)=d(x)+n(x)
$$

また(44)式を(35)式に代入して， 


$$
\rho^{2}(x)=n(x) / d(x)
$$

となる. $n_{l}(x)$ が(27) で与えられることは分母の場合と 同様にして容易に確かめられる。

定理 1 についていくつかコメントしておく. はじめに $\rho^{2}(x)$ の分母, 分子多項式の次数について述べる. チェビ シェフ多項式 $U_{i}(x)$ は $i$ 次多項式であるから, 分子多項 式の次数は一般に $2(n-1)$ 次である. しかし分母多項式 $d(x)$ については, その次数は $2(n-2)$ 次となる. 実際, $d_{l_{1} l_{2}}(x)$ の $U_{n-1}(x)$ に対する係数は

$$
A\left(\begin{array}{cc}
1 & n+1 \\
l_{1} & l_{2}
\end{array}\right)=\left|\begin{array}{ll}
a_{0 l_{1}} & a_{0 l_{2}} \\
a_{n l_{1}} & a_{n l_{2}}
\end{array}\right|
$$

であるが,これは(19)より零に等しい.この分母におけ る次数低下は (1)で最高次係数を固定したことによるも のであることに注意しておく。

つぎに文献 1)で取り上げられた特性多項式

$$
f(z, \boldsymbol{p})=z^{n}+p_{n} z^{n-1}+\cdots+p_{2} z+p_{1}
$$

の場合の公式を導いておく．このとき $k=n$ であり,

$$
\boldsymbol{A}=\left[\begin{array}{cccc}
1 & & & \\
& 1 & & \\
& & \ddots & \\
& & & 1 \\
0 & 0 & \cdots & 0
\end{array}\right] \in R^{(n+1) \times n}, \boldsymbol{a}^{0}=\left[\begin{array}{c}
0 \\
0 \\
\vdots \\
0 \\
1
\end{array}\right] \in R^{(n+1)}
$$

となる.また $n \geq 2$ のとき，仮定 $1,2,3$ が成り立つこと は容易に確かめられる。したがって定理 1 が適用でき, 直接的な計算によりつぎの系を得る。

《系1》 $n \geq 2$ のとき

$$
\rho=\min \left\{\mu_{1}, \mu_{2}, \bar{\rho}\right\}
$$
ただし

$$
\begin{aligned}
& \mu_{1}=\left|p_{1}^{0}+p_{2}^{0}+\cdots+p_{n}^{0}+1\right| / \sqrt{n} \\
& \mu_{2}=\left|p_{1}^{0}-p_{2}^{0}+\cdots+(-1)^{n-1} p_{n}^{0}+(-1)^{n}\right| \sqrt{n} \\
& \bar{\rho}^{2}=\inf _{x \in(-1,1)} \rho^{2}(x) \\
& \rho^{2}(x)=\frac{\left\|\boldsymbol{U}(x) \boldsymbol{\delta}^{0}\right\|^{2}}{(n-1) U_{0}(x)^{2}+(n-2) U_{1}(x)^{2}+\cdots+U_{n-2}(x)^{2}}
\end{aligned}
$$

$$
\text { ここで } \boldsymbol{U}(x) \in R^{n \times(n+1)} \text { はつぎで与えられる. }
$$

$$
\begin{gathered}
{\left[\begin{array}{ccc}
0 & U_{0}(x) & U_{1}(x) \\
-U_{0}(x) & 0 & U_{0}(x) \\
-U_{1}(x) & -U_{0}(x) & 0 \\
\vdots & & \\
-U_{n-2}(x) & -U_{n-3}(x) & -U_{n-4}(x) \\
\cdots & U_{n-2}(x) & U_{n-1}(x) \\
& U_{n-3}(x) & U_{n-2}(x) \\
& U_{n-4}(x) & U_{n-3}(x) \\
\ddots & & \vdots \\
\cdots & 0 & U_{0}(x)
\end{array}\right]}
\end{gathered}
$$

また

$$
\boldsymbol{\delta}^{0}=\left[\boldsymbol{p}^{0 T}: 1\right]^{T}
$$

である。

最後に $\boldsymbol{a}=\left[a_{n 1} \cdots a_{n k}\right]^{T} \neq \mathbf{0}$ の場合，すなわち最高次係 数も捸動を受ける場合について検討しておく.このとき，

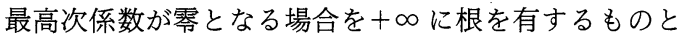
して不安定多項式のクラスにまとめると, $\boldsymbol{p}^{0}$ から

$$
\Pi_{\infty}=\left\{\boldsymbol{p}: a_{n 1} p_{1}+\cdots+a_{n k} p_{k}+a_{n 0}=0\right\}
$$

への距離

$$
\mu_{\infty}=\left|\boldsymbol{\alpha}^{T} \boldsymbol{p}^{0}+1\right| /\|\boldsymbol{\alpha}\|
$$

を考慮して(21)式を

$$
\rho=\min \left\{\mu_{\infty}, \mu_{1}, \mu_{2}, \bar{\rho}\right\}
$$

と修正するだけで，定理 1 は依然として成り立つ。ただ し, $\rho^{2}(x)$ の分母の次数は一般に分子の次数と同じく $2(n-1)$ 次となる.

\section{3. 検 討}

定理 1 より, 最大安定超球の半径 $\rho$ の計算は, 実質的 に $\bar{\rho}$ の計算に帰着する. 有理関数 $\rho^{2}(x)$ の区間 $(-1,1)$ における最小值を求める問題はその分母・分子多項式へ の代数演算によって容易に解ける. しかしながら仮定 1 , 2,3 が成り立たない問題ではこの方法では誤った結果を 与える可能性がある。すなわち代数演算で得られた最小 值を $\rho^{*}$ とすると, $\bar{\rho}<\rho^{*}$ となる場合がある.この点は文 献 2)，3，9）ではあまり強調されていなかった問題であ る.

本節では, 仮定 $1,2,3$ が成立しない場合にも関数 $\rho^{2}(x)$ の分子の実根を求めることにより, 同様の手順で 半径 $\rho$ が計算可能であることを示す.

さて $f\left(e^{j \theta}, \boldsymbol{p}\right)=0(0 \leq \theta \leq \pi)$ の条件 (15) (20)を $\boldsymbol{p}=\boldsymbol{p}^{0}$ $+\delta \boldsymbol{p}, \boldsymbol{\delta}^{0}=\boldsymbol{A} \boldsymbol{p}^{0}+\boldsymbol{a}^{0}$ を用いてつぎのように書き直して おく.

$$
D A \delta p+D \delta^{0}=0
$$

また

$$
\boldsymbol{e}_{i}^{T} \boldsymbol{A} \delta \boldsymbol{p}+\boldsymbol{e}_{i}^{T} \boldsymbol{\delta}^{0}=0, \quad i=1,2
$$

公称パラメータ $\boldsymbol{p}^{0}$ は安定領域にあるものと仮定したの で, $f\left(e^{j \theta}, \boldsymbol{p}^{0}\right) \neq 0, \forall \theta \in[0, \pi]$ ，すなわち

$$
D \boldsymbol{\delta}^{0} \neq \mathbf{0}
$$

また

$$
\boldsymbol{e}_{i}^{T} \boldsymbol{\delta}^{\mathbf{0}} \neq 0, \quad i=1,2
$$

であることに注意する。

以上の準備の下で仮定 1 が，ある $x \in(-1,1)$ に対して 不成立の場合を行列 $\boldsymbol{D A}$ と $\boldsymbol{D B}=\left[\boldsymbol{D A}: D \boldsymbol{\delta}^{0}\right]$ のラン ク条件により，つぎの三つの場合にわけて検討する。

i ） $\operatorname{rank} \boldsymbol{D A}=1$ かつ $\operatorname{rank} \boldsymbol{D B}=1$ の場合

このとき $(56)$ は解を有し, $\Pi(x)$ は $k-1$ 次元アフィン 
部分空間をなす. 行列 $\boldsymbol{D A}$ は仮定より非零行ベクトルを 有するので, その一つを取り出して(ここでは第 $i$ 行べク トルとする)

$$
\Pi(x)=\left\{\boldsymbol{p}^{0}+\delta \boldsymbol{p}: \boldsymbol{d}_{i}(x)^{T} \boldsymbol{A} \delta \boldsymbol{p}+\boldsymbol{d}_{i}(x)^{T} \boldsymbol{\delta}^{0}=0\right\}(60)
$$

と表現できる。したがって,この場合にはつぎを得る.

$$
\rho(x)=\left|\boldsymbol{d}_{i}(x)^{T} \boldsymbol{\delta}^{0}\right| /\left\|\boldsymbol{d}_{i}(x)^{T} \boldsymbol{A}\right\|
$$

ii ） $\operatorname{rank} \boldsymbol{D A}=1$ かつ rank $\boldsymbol{D B}=2$ の場合

このとき $(56)$ には解がなく,したがって $\rho(x)=+\infty$.

iii） $\operatorname{rank} \boldsymbol{D A}=0$ の場合

(58)より（56）は解をもたない. ゆえに $\rho(x)=+\infty$ i )の条件を満たす点 $x$ を拡大点, また ii ), iii)の条件を 満たす点 $x$ を消失点と呼ぶ. $x= \pm 1$ は離散時間系の場 合，常に拡大点または消失点となる。これに対して $x$ $\in(-1,1)$ の拡大点は係数デー夕に依存する一時的なも のと考えられる.

つぎに拡大点および消失点の求め方について考える. はじめにランク条件 $\operatorname{rank} \boldsymbol{D A}<2$ は $d(x)=0$ と等価で あることに注意する．すなわち仮定 1 が不成立となるの は $d(x)$ の根においてであり，またその場合に限る。

同様に条件 rank $\boldsymbol{D B}<2$ は $\tilde{n}(x)=0$ と等価である.ま た(58)より,すべての $x \in(-1,1)$ に対して, $\operatorname{rank} \boldsymbol{D B}$ $\geq 1$. したがって $\tilde{n}(x)=0$ は $x$ において, 行列 $\boldsymbol{D B}$ のラ ンクが 1 であることと等価であることがわかる.

ここで後の便宜のために, 極小条件から導かれる非負 值多項式 $q(x)(q(x) \geq 0, \forall x \in R)$ の性質をまとめてお (11).

事実 $1 . q(x)$ が非負値ならば，その実根の多重度 は常に偶数である.

事実 2. $q_{1}(x), q_{2}(x)$ が非負值かつ, $q_{1}(x) \geq q_{2}(x)$, $\forall x \in R$ とする.このとき $x_{0} \in R$ が $q_{1}$ の $n_{1}$ 重根で あるならば, $x_{0}$ は $q_{2}$ の $n_{2}$ 重根である.ただし $n_{2} \geq n_{1}$ 。 明らかに $d(x)$ と $\tilde{n}(x)$ は非負值多項式であり, $d(x)$ $\leq \tilde{n}(x)$ を満たしている。 したがって事実 2 より， $\tilde{n}(x)$ の根は多重度を含めてすべて $d(x)$ の根となることがわ かる.これより, 拡大点を求めるには区間 $(-1,1)$ にある $\tilde{n}(x)$ の根の中から, $\boldsymbol{D A} \neq 0$ を満たすものをとればよ い. また区間 $(-1,1)$ にある $d(x)$ の根であり, 拡大点で ないものはすべて消失点となる.

上で述べた結果は $\tilde{n}(x)$ を $n(x)$ に置き換えて, そのま ま成り立ちつぎの定理にまとめられる。

《定理 2》区間 $(-1,1)$ における $n(x)$ の根を $\alpha_{1}, \cdots$, $\alpha_{s}$ とし, その多重度を $2 h_{1}, \cdots, 2 h_{s}$ とする.ここで $i=1$, $\cdots, s$ に対して，つぎの数を定義する.

$$
\mu_{i}= \begin{cases}\left|\boldsymbol{d}_{i}\left(\alpha_{i}\right)^{T} \boldsymbol{\delta}^{0}\right| /\left\|\boldsymbol{d}_{j}\left(\alpha_{i}\right)^{T} \boldsymbol{A}\right\| \cdots \boldsymbol{D}\left(\alpha_{i}\right) \boldsymbol{A} \neq 0 \\ +\infty & \cdots \boldsymbol{D}\left(\alpha_{i}\right) \boldsymbol{A}=0\end{cases}
$$

$$
\begin{aligned}
& \text { また同様に } i=1,2 \text { に対して }
\end{aligned}
$$

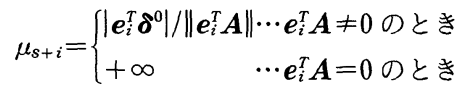

を定義するこことを

$$
\rho=\min \left\{\mu_{1}, \cdots, \mu_{s}, \mu_{s+1}, \mu_{s+2}, \rho^{*}\right\}
$$
ただし

$$
\begin{aligned}
& \left(\rho^{*}\right)^{2}=\inf _{x \in(-1,1)} \frac{n_{0}(x)}{d_{0}(x)} \\
& d_{0}(x)=d(x) /\left(x-\alpha_{1}\right)^{2 h_{1}} \cdots \cdots\left(x-\alpha_{s}\right)^{2 h_{s}} \\
& n_{0}(x)=n(x) /\left(x-\alpha_{1}\right)^{2 h_{1}} \cdots \cdots\left(x-\alpha_{s}\right)^{2 h_{s}}
\end{aligned}
$$

（証明）前に検討したことから， $n(x)$ と $\tilde{n}(x)$ は $(-1,1)$ において重複度を含めて同じ根分布をもつこと を示せば証明が終わる。

$\tilde{n}(x)$ の実根は多重度を含めてすべて $d(x)$ の根とな ること,および $n(x)=\tilde{n}(x)-d(x)$ より, $\tilde{n}(x)$ の実現は $n(x)$ の根でもあり, その多重度は $\tilde{n}(x)$ に対する多重度 と等しい.したがって区間 $(-1,1)$ における $n(x)$ の根は すべて $\tilde{n}(x)$ の根でもあることを示せばよい. $n(s)=0$, $s \in(-1,1)$ かつ $\tilde{n}(s)>0$ として矛盾を示す.第 2 の条件 より, $\boldsymbol{D} \boldsymbol{A} \boldsymbol{A}^{T} \boldsymbol{D}^{T}>0$. また第 1 の条件より, $\tilde{n}(s)=d(s)$, したがって $\boldsymbol{D} \boldsymbol{B} \boldsymbol{B}^{T} \boldsymbol{D}^{T}=\boldsymbol{D} \boldsymbol{A} \boldsymbol{A}^{T} \boldsymbol{D}^{T}>0$ を得る．これから $\boldsymbol{D}^{0}=0$ を得るが，これは(58)式に矛盾する。(証明終)

$n_{0}(x)$ は $(-1,1)$ に根をもたず, したがって $d_{0}(x)$ と同 区間に共通根をもつことはない.したがって (65)式の計 算には, 商の導関数公式から得られる極值条件

$$
n_{0}(x) d_{0}^{\prime}(x)-n_{0}^{\prime}(x) d_{0}(x)=0
$$

より, $(-1,1)$ 上の根 $s_{i}$ を求め, $n_{0}\left(s_{i}\right) / d_{0}\left(s_{i}\right)$ の最小値を 求めればよい.ただし $d_{0}^{\prime}(x), n_{0}^{\prime}(x)$ はそれぞれ $d_{0}(x)$, $n_{0}(x)$ の導関数を表わす.また当然ながら $\mu_{i} \neq+\infty$ なる $\alpha_{i}$ が拡大点である。

\section{4. 例 題}

これまでの同種の研究においては，定理 1 のように三 つの数值の最小値として最大安定超球の半径を与えるこ とで満足していたように思われる。これは, 係数パラメ ー夕空間における結果と同じ形のものであるが, より一 般にアフィンパラメータを対象とする場合には，これで は誤った結果を導く可能性がある，これは拡大点が存在 すれば, その点 $x$ において真の $\rho(x)$ の值が $\rho(x)$ の連続 性から想定される值よりも一般に小さくなるからであ る. 実際に数值例を与える.

(例 1 )

$$
\begin{aligned}
f(z, \boldsymbol{p})= & z^{4}+\left(p_{1}+0.3\right) z^{3}+\left(p_{2}+0.5\right) z^{2} \\
& +\left(p_{1}+p_{2}-0.2\right)
\end{aligned}
$$

このとき

ただし $j$ は $\boldsymbol{D}\left(\alpha_{i}\right) \boldsymbol{A}$ の非零行を指す。 
$\boldsymbol{D}=\left[\begin{array}{ccccc}1 & x & 2 x^{2}-1 & 4 x^{3}-3 x & 8 x^{4}-8 x^{2}+1 \\ 0 & 1 & 2 x & 4 x^{2}-1 & 8 x^{3}-4 x\end{array}\right]$

$\boldsymbol{A}=\left[\begin{array}{ll}1 & 1 \\ 0 & 0 \\ 0 & 1 \\ 1 & 0 \\ 0 & 0\end{array}\right], \quad \boldsymbol{a}^{0}=\left[\begin{array}{c}-0.2 \\ 0 \\ 0.5 \\ 0.3 \\ 1\end{array}\right]$

ここで $\boldsymbol{p}^{\mathbf{0}}=\left[\begin{array}{ll}0 & 0\end{array}\right]^{T}$ としてつぎを得る。

$$
\begin{aligned}
& d(x)=4 x^{2}(1-2 x)^{2} \\
& n(x)=x^{2}\left(128 x^{4}+51.2 x^{3}-52.16 x^{2}\right.
\end{aligned}
$$$$
-13.44 x+9.36)
$$

$d(x)$ の $(-1,1)$ 上の根は 0 と 0.5 の二つであるが, $n(x)$ $=0$ の同区間上の根は 0 のみである。ゆえに一時的な拡 大点は 0 のみで, 0.5 は消失点となる。このとき $\rho(x)$ は Fig. 1 のようになる。最大安定半径は $x=0$ で生じ， $\rho$ $=0.3$ となる.また $x=-0.606$ で $\rho^{*}=0.464$ となる. Fig. 2 にパラメータ平面上の安定領域を示す。Fig. 1, Fig. 2 で○は曲線の連続性から想定された $x=0$ の点を表わ す. $x=0$ のとき, 正しくは Fig. 2 では○をとおる垂直線 となり，Fig. 1 では○で示された点となる。

一般に $d(x)$ の根の多重度から, 行列 $\boldsymbol{D}(x) \boldsymbol{A}$ のラン

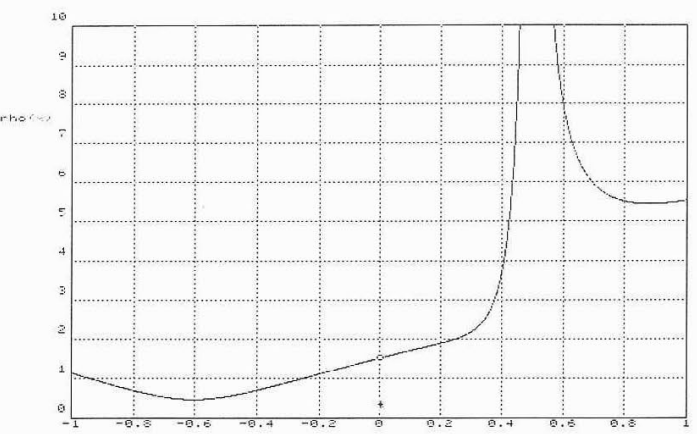

Fig. $1 \rho(x)$ for Example 1

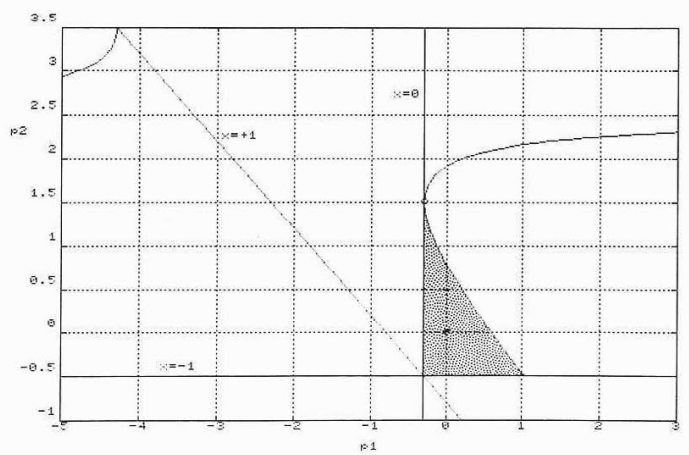

Fig. 2 Stability region for Example 1
クを知ることはできない。例 1 では $x=0$ でランクが 1 , 多重度が 2 であったが，同じくランクが 1 であっても 4 重根という場合も存在する。つぎの例はそのような例で ある。

(例 2)

$$
\begin{aligned}
f(z, \boldsymbol{p})= & z^{4}+\left(p_{1}+3 p_{2}+0.3\right) z^{3}+\left(p_{1}+p_{2}+1.1\right) z^{2} \\
& +\left(p_{1}+2 p_{2}+0.2\right) z+\left(p_{1}+2 p_{2}+0.2\right)
\end{aligned}
$$

の場合を考えると

$$
d(x)=16 x^{4}
$$

すなわち $x=0$ を 4 重根とするが， $\boldsymbol{D}(0) \boldsymbol{A}=\left[\begin{array}{rr}0 & 1 \\ 0 & -1\end{array}\right]$ の ランクは 1 である、 $\boldsymbol{p}^{0}=\left[\begin{array}{ll}0 & 0\end{array}\right]^{T}$ とすると

$$
n(x)=x^{2}\left(320 x^{4}+326.4 x^{3}+19.36 x^{2}-32 x+4\right)
$$

$x=0$ は $n(x)$ の根でもあるので拡大点となる.最大安定 半径は $x=0$ で生じ $\rho=0.1$ となる。また $x=-0.665$ で $\rho^{*}=0.248$ となる. Fig. 3 に $\rho(x)$, Fig. 4 に安定領域を 示す.Fig. 3 で $x=0, \rho=0.1$ の点はので示されている。

最後の例は極端なケースとして分母 $d(x) \equiv 0$ という 場合が起こり得ることを示す。

(例 3 )

$$
f(z, \boldsymbol{p})=z^{4}+\left(p_{1}+0.5\right) z^{3}+\left(p_{2}+0.5\right) z^{2}+p_{2} z+p_{1}
$$
このとき

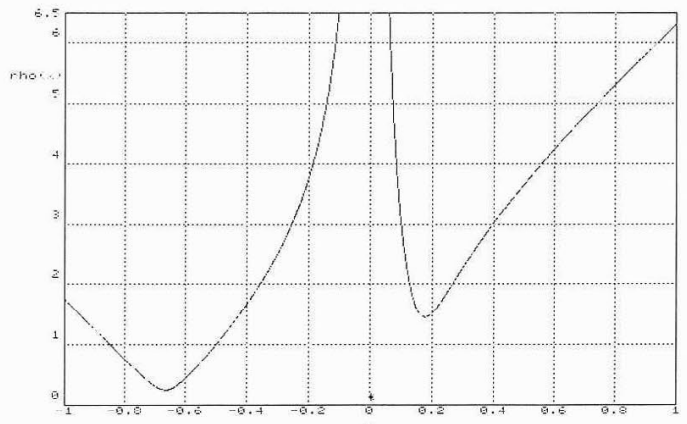

Fig. $3 \rho(x)$ for Example 2

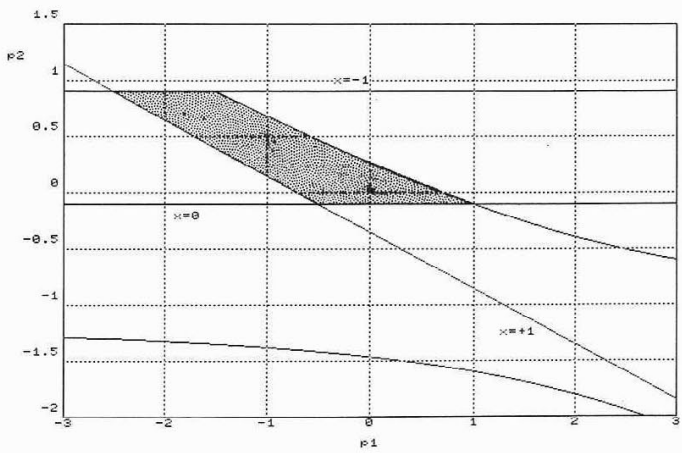

Fig. 4 Stability region for Example 2 


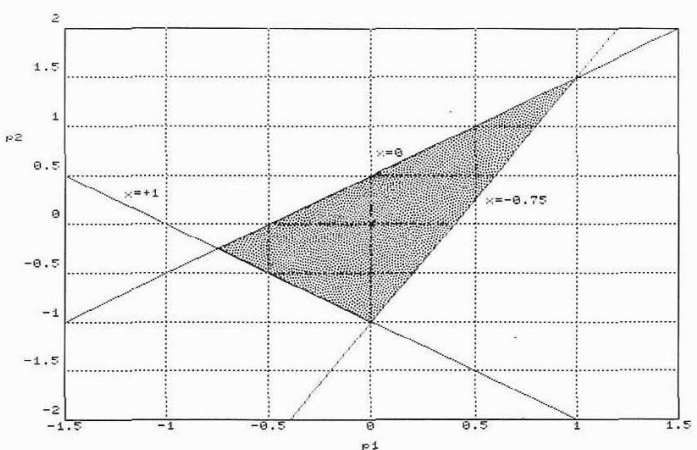

Fig. 5 Stability region for Example 3

$$
\boldsymbol{D}(x) \boldsymbol{A}=\left[\begin{array}{cc}
(2 x-1)\left(2 x^{2}+x-1\right) & 2 x^{2}+x-1 \\
(2 x-1)(2 x+1) & 2 x+1
\end{array}\right]
$$

より $d(x) \equiv 0$ となる。また詳しくは $\boldsymbol{D}(x) \boldsymbol{A}$ のランクは 恒等的に 1 となっている。したがって $n(x)$ のすべての 根が拡大点となり，その他の $x$ についてはすべて消失点 となる。 $p^{0}=\left[\begin{array}{ll}0 & 0\end{array}\right]^{T}$ とすると

$$
n(x)=32 x^{2}(x+3 / 4)^{2}\left(2 x^{2}-2 x+1\right)
$$

により $x=0, x=-3 / 4$ が拡大点である.また $x=-1$ は 消失点となる。最大安定半径は $x=0$ で生じ $\rho=\sqrt{2} / 4$ と なる. Fig. 5 に安定領域を示す。

\section{5.おわりに}

本稿では特性多項式の係数が，複数パラメータのアフ イン関数で与えられている離散時間系を考え，パラメー 夕空間における最大安定超球の半径 $\rho$ を与える二つの 簡潔な公式を与えた。その 1 つは文献 1)で扱われたよう な係数パラメータの場合と平行した結果を与えるもの で, このとき $\rho$ の計算は, 二つの多項式 $n(x), d(x)$ の比 の最小を求めることに帰着する。公式によると，これら の多項式はチェビシェフ多項式を用いることにより，ア フィン係数データより容易に得られるものである。

第 2 の公式は係数パラメータの場合との相違点, すな わち 3 個以上の拡大点の存在可能性を考慮したものであ る. 公式によると, 拡大点の存在は多項式 $n(x)$ の根を調 ベることにより，容易に判別可能である。

$x= \pm 1$ 以外の拡大点は, 係数データの微少な変動によ って消滅する。したがって, 最大安定超球の半径がこの ような拡大点で達せられている場含, データの変動によ つて安定余裕が不連続的に変化する場合が予想される。 連続時閒系に対して, Barmish ら ${ }^{12)} ゃ V$ icino と Tesi ${ }^{6)}$ が与えた例は同様の事情のもとで生じたものと考えられ る.このような不連続変化を生ずるための条件には理論 的な興味がもたれるが，今後の課題として残される。

\section{参 考 文 献}

1) C. B. Soh, C. S. Berger and K. P. Dabke: On the Stability Properties of Polynomials with Perturbed Coefficients, IEEE Trans. Automatic Control, AC-3010, 1033/1036 (1985)

2) R. M. Biernacki, H. Hwang and S. P. Bhattacharyya : Robust Stability with Structured Real Parameter Perturbations, IEEE Trans., AC-32-6, 495/505 (1987)

3) H. Chapellat, S. P. Bhattacharyya and L. H. Keel : Stability Margins for Hurwitz Polynomials, Proc. 27th IEEE Conf. on Decision and Control, 1392/1398 (1988)

4) A. Vicino: Some Results on Robust Stability of Discrete-time Systems, IEEE Trans., AC-33-9, 844/847 (1988)

5) T.E. Djaferis: The Largest Stability Hypercube for Families of Polynomials with Linear Uncertainty, Proc. 1989 American Control Conf., 633/638 (1989)

6) A. Vicino and A. Tesi: Regularity Conditions for Robust Stability Problems with Linearly Structured Perturbations, Proc. 29th IEEE Conf. on Decision and Control, 46/51 (1990)

7) Y.Z. Tsypkin and B. T. Polyak: Frequency Domain Criteria for $l^{p}$-robust Stability of Continuous Linear Systems, Institute for Control Sciences, Moscow, USSR (1990)

8）上家, 古田：離散・線形システムにおける $l^{p}$-ロバスト安 定性の判別法, 計測自動制御学会論文集, 27-6, 628/ 632 (1991)

9) G. Aguirre, H. Chapellat and S. P. Bhattacharyya : Stability Margins for Discrete-time Uncertain Systems, Proc. 28th IEEE Conf. on Decision and Control, 1874/1875 (1989)

10）森, 小亀：構造的不確かさをもつ制御系の安定解析, シス テム/制御/情報, 37-5，268/277（1991）

11）高木貞治：解析概論，岩波書店（1938）

12) B. R. Barmish, P. P. Khargoneckar, Z. C. Soh and R. Tempo: Robustness Margin Need Not Be a Continuous Function of the Problem Data, Systems \& Control Letters, 15-2, 91/98 (1990)

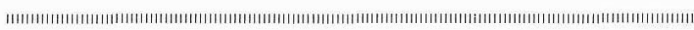

$$
\text { [著 者 紹 介] }
$$

\section{小亀 英 已(正会員)}

1973 年, 京都大学大学院博士課程単位取 得退学 (電気工学専攻). 同年, 京都大学工 学部助手. 1980 年大阪工業大学講師, 現在 同教授。制御系の解析・設計の研究に従事。 京都大学工学博士. システム制御情報学会 の会員.

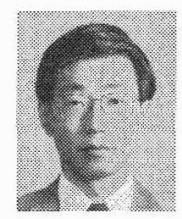

\section{吉田誠一}

1991 年, 大阪工業大学工学部電気工学科 卒業, 現在, 同大学人学院工学研究科修士 課程電気工学専攻在学中。不確定システム のロバスト安定性について研究中。

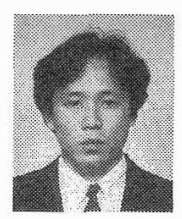



武 宏 (正会員)

1974 年, 京都大学大学院博士課程単位取 得退学 (電気工学専攻). 同年京都大学工学 部助手, 同助教授を経て, 1990 年 6 月より 京都工芸瀻維大学教授. 制御系解析の研究 に従事. 京都大学工学博士. システム制御 情報学会の会員.

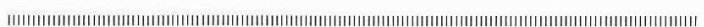

